\section{Diagnóstico ambiental de empresas y negocios aledaños a Los Pantanos de Villa (urbanización Huertos de Villa)}

Jhanaly Lady Noelia Fernández-Morales ${ }^{1 *}{ }^{\oplus}$, Janet Angelica Leyva-Quispe ${ }^{2 \oplus}$ y Sheimy Yahaira Quiroz-Quispe ${ }^{3 \odot}$
Environmental diagnosis of companies and businesses in the vicinity of Los Pantanos de Villa wetlands (Huerto de Villa development)
1 Facultad de Ingeniería, Universidad Privada del Norte. Lima, Perú.

2 Facultad de Ingeniería y Gestión, Universidad Nacional Tecnológica de Lima Sur. Lima, Perú.

3 Facultad de Ingeniería y Arquitectura, Universidad César Vallejo. Lima, Perú.

\section{SOUTH} SUSTAINABILITY

Citar como: Fernández-Morales, J. L. N. et al. (2021). «Diagnóstico ambiental de empresas y negocios aledaños a Los Pantanos de Villa (urbanización Huertos de Villa)». South Sustainability, 2(2), e035. DOI: 10.21142/SS-0202-2021-e001

Artículo recibido: 25/10/2021 Revisado por pares

Artículo aprobado: 27/12/2021

\section{(c) (i)}

(c) Las autoras, 2021. Publicado por la Universidad Científica del Sur (Lima, Perú)

${ }^{*} E$-mail de correspondencia: noeliafer.103@gtmail.com

\section{RESUMEN}

El presente estudio se realizó en la urbanización Huertos de Villa, considerada una zona urbanaindustrial, ubicada en el límite norte del Refugio de Vida Silvestre Los Pantanos de Villa. El objetivo del estudio fue realizar un diagnóstico ambiental de las empresas y los negocios aledaños a este humedal. Por ello, primero se identificó el número total de empresas y negocios en la zona de estudio; seguidamente, se determinó la existencia de planes de gestión ambiental y el estado situacional de cada uno de ellos mediante la aplicación de encuestas respecto a sus prácticas ambientales. Como resultado se identificó la existencia de 16 empresas formales y 9 negocios informales; además, se concluyó que solo el $33 \%$ de las empresas encuestadas cuentan con un instrumento de gestión ambiental.

Palabras clave: diagnóstico ambiental, plan, humedal, estado situacional

ABSTRACT

This study was conducted in the Huertos de Villa development, considered an urban-industrial area located on the northern edge of Los Pantanos de Villa Wildlife Refuge. The aim of the study was to conduct an environmental diagnosis of the companies and businesses surrounding the wetland. First, the total number of companies and businesses existing in the study area was identified; then, the existence of management plans and the status of each was determined, through the application of environmental surveys focused on their environmental practices. As a result, 16 formal companies and 9 informal businesses were identified. Furthermore, it was determined that only $33 \%$ of the companies surveyed possess an environmental management instrument.

Keywords: environmental diagnosis, plan, wetland, situational status 


\section{Introducción}

Las actividades industriales generan graves problemas ambientales debido al agotamiento de varios recursos no renovables y el uso excesivo consumo de energía (Pacheco Bustos et al., 2017). Por ello, la presencia de industrias y negocios ocasionan los principales deterioros en el medioambiente.

Pongamos por caso a Noruega, país líder en la producción de salmón, lo que ha provocado un impacto negativo en la especie $y$, además, ha afectado a otras especies, como la trucha marina y las pesquerías costeras (Olaussen, 2018). Algo semejante ocurre en China, debido a que se convirtió en el principal pilar en la industria de la acuicultura, lo que ocasiona un desequilibrio en el ecosistema (He et al., 2016).

En el Perú se manifestó una situación similar en el distrito de Chorrillos (provincia de Lima), donde se encuentra el Refugio de Vida Silvestre Los Pantanos de Villa (RVSPV), que enfrenta una contaminación antrópica (León Sulca, 2019) causada por las empresas industriales ubicadas a su alrededor. En consecuencia, se generan algunos impactos negativos en el humedal y su biodiversidad.

Los autores del presente artículo plantearon como objetivo principal realizar un diagnóstico ambiental de las empresas y negocios aledaños al RVSPV, en la urbanización Huertos de Villa, con el fin de conocer si cuentan con una buena planificación y gestión ambiental.

\section{Materiales y métodos}

\section{Área de estudio}

La urbanización Huertos de Villa se ubica en el distrito de Chorrillos, en la zona costera de Lima, Perú (ver figura 1). Esta urbanización se considera como una zona urbana (Ramirez et al., 2018) y abarca una extensión de 263,27 hectáreas (Pulido, 2018). Los Pantanos de Villa fueron declarados zona reservada en 1989 por el Ministerio de Agricultura (Solano y Chirinos, 2001), debido a que albergan distintas especies de flora y fauna.

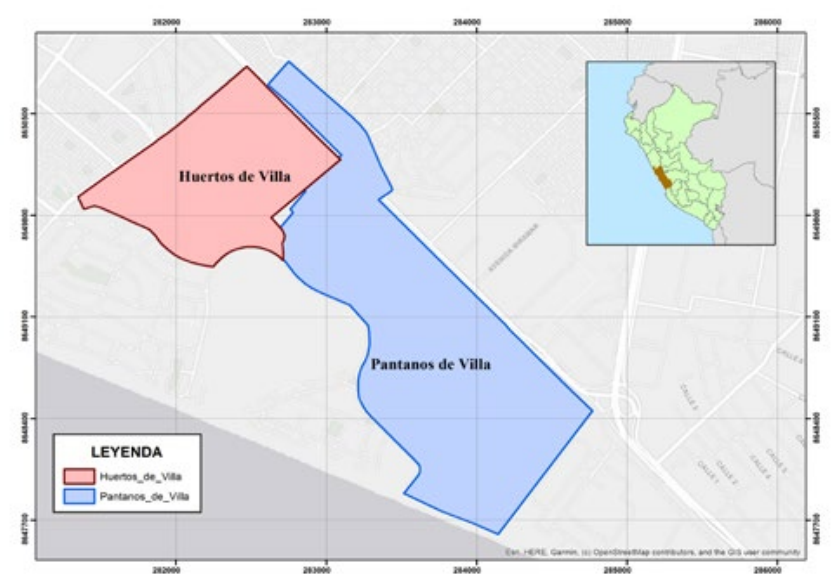

Figura 1. Ubicación de la urbanización Huertos de Villa. Fuente: Elaboración propia.

\section{Población y muestra}

La población que se estudió está constituida por 16 empresas y 9 negocios formales e informales aledaños al RVSPV. Fueron identificados mediante la primera salida a campo y la manipulación del aplicativo GPS Essentials a través de coordenadas (ver figura 2).

A partir de la segunda salida a campo, se obtuvo una muestra de entidades privadas de 6 empresas y 7 negocios, a través de una encuesta virtual que buscaba conocer si presentaban un comportamiento ambiental en las actividades realizadas. Con las empresas que no aceptaron realizar la encuesta se desarrolló una descripción de sus actividades basada en la salida de campo.

A continuación, la tabla 1 presenta la lista de empresas y negocios determinados como población.

Tabla 1. Datos de las empresas y negocios de la urbanización Huertos de Villa considerados en el estudio.

\begin{tabular}{|c|c|c|c|c|}
\hline $\mathbf{N} .^{\circ}$ & Entidades & RUC & $\begin{array}{c}\text { Rubro de la } \\
\text { organización }\end{array}$ & Encuesta \\
\hline \multicolumn{5}{|c|}{ EMPRESAS } \\
\hline 1 & Wenam EIRL & 20178313488 & $\begin{array}{l}\text { Elaboración } \\
\text { de productos } \\
\text { alimenticios }\end{array}$ & Sí \\
\hline 2 & $\begin{array}{c}\text { Comercializadora } \\
\text { S\&E SA }\end{array}$ & 20511535612 & $\begin{array}{c}\text { Construcción de } \\
\text { edificios }\end{array}$ & Sí \\
\hline 3 & Serpanmin SAC & 20518179587 & $\begin{array}{c}\text { Fabricación } \\
\text { de maquinaria } \\
\text { minera }\end{array}$ & Sí \\
\hline 4 & Iglú SAC & 20419665992 & $\begin{array}{c}\text { Fabricación } \\
\text { de productos } \\
\text { mineros no } \\
\text { metálicos NCP }\end{array}$ & Sí \\
\hline 5 & Sabadi & 20549264566 & $\begin{array}{c}\text { Mantenimiento } \\
\text { y reparación de } \\
\text { vehículos }\end{array}$ & Sí \\
\hline 6 & KPA SAC & 20202971157 & $\begin{array}{c}\text { Venta de } \\
\text { maquinaria, } \\
\text { equipo y } \\
\text { materiales }\end{array}$ & Sí \\
\hline 7 & TDM Group & 201235313889 & $\begin{array}{c}\text { Ferretería y } \\
\text { acabados }\end{array}$ & No \\
\hline 8 & $\begin{array}{c}\text { SOLID - Servicios } \\
\text { Generales LID } \\
\text { SAC }\end{array}$ & 20517953262 & $\begin{array}{l}\text { Vehículos, } \\
\text { servicio, } \\
\text { autopartes }\end{array}$ & No \\
\hline 9 & Las Caleras & 20452614767 & $\begin{array}{l}\text { Alimentos } \\
\text { elaborados }\end{array}$ & No \\
\hline 10 & $\begin{array}{c}\text { Construredes } \\
\text { SAC }\end{array}$ & 20518509391 & $\begin{array}{c}\text { Instalaciones de } \\
\text { gas natural }\end{array}$ & No \\
\hline 11 & $\begin{array}{c}\text { Globenatural } \\
\text { Internacional SA }\end{array}$ & 20382056681 & $\begin{array}{c}\text { Empresa de } \\
\text { manufacturas }\end{array}$ & No \\
\hline 12 & Best Brants & 20508766751 & $\begin{array}{l}\text { Alquiler y venta } \\
\text { de andamios }\end{array}$ & No \\
\hline
\end{tabular}




\begin{tabular}{|c|c|c|c|c|}
\hline 13 & Ovosur SA & 20344506842 & $\begin{array}{l}\text { Elaboración } \\
\text { de producto } \\
\text { alimenticios }\end{array}$ & No \\
\hline 14 & Zender SAC & 20510256361 & $\begin{array}{c}\text { Venta de } \\
\text { materiales de } \\
\text { construcción }\end{array}$ & No \\
\hline 15 & $\begin{array}{c}\text { Grupo Vivargo } \\
\text { SAC }\end{array}$ & 20508669802 & $\begin{array}{l}\text { Autotransporte } \\
\text { de carga general }\end{array}$ & No \\
\hline 16 & $\begin{array}{c}\text { Andamios Altos } \\
\text { SAC }\end{array}$ & 20392559443 & $\begin{array}{l}\text { Alquiler de } \\
\text { maquinarias y } \\
\text { equipo }\end{array}$ & No \\
\hline \multicolumn{5}{|c|}{ NEGOCIOS } \\
\hline 1 & $\begin{array}{l}\text { Automotriz } \\
\text { Sobre Ruedas }\end{array}$ & - & $\begin{array}{l}\text { Lavado de } \\
\text { vehículos }\end{array}$ & Sí \\
\hline 2 & Car wash & - & $\begin{array}{l}\text { Lavado de } \\
\text { vehículos }\end{array}$ & Sí \\
\hline 3 & Ricardo Motor & - & $\begin{array}{l}\text { Reparación de } \\
\text { vehículos }\end{array}$ & Sí \\
\hline 4 & $\begin{array}{c}\text { Noemi } \\
\text { Chatarrería }\end{array}$ & - & $\begin{array}{l}\text { Recolección de } \\
\text { residuos sólidos } \\
\text { inorgánicos }\end{array}$ & Sí \\
\hline 5 & $\begin{array}{l}\text { Planchado y } \\
\text { pintura }\end{array}$ & - & $\begin{array}{l}\text { Lavado de } \\
\text { vehículos }\end{array}$ & Sí \\
\hline 6 & Baterías Fulgor & - & $\begin{array}{l}\text { Reparación de } \\
\text { vehículos }\end{array}$ & Sí \\
\hline 7 & $\begin{array}{l}\text { Estructuras } \\
\text { metálicas }\end{array}$ & - & $\begin{array}{l}\text { Fabricación } \\
\text { de productos } \\
\text { metálicos }\end{array}$ & Sí \\
\hline 8 & Ergisa Perú & - & $\begin{array}{l}\text { Fabricación } \\
\text { de productos } \\
\text { metálicos }\end{array}$ & No \\
\hline 9 & Chatarrería & - & $\begin{array}{l}\text { Recolección de } \\
\text { residuos sólidos } \\
\text { inorgánicos }\end{array}$ & No \\
\hline
\end{tabular}

\section{Instrumentos}

Se manipuló la variable «diagnóstico ambiental» con la ejecución de dos encuestas de respuesta dicotómica para la recolección de datos, una para empresas y otra para negocios, mediante formularios de Google. A la vez, se aplicó un análisis de confiabilidad de Kuder y Richardson (KR 20), que es un instrumento de fiabilidad para la encuesta (Juárez y Tobón, 2018). El instrumento fue validado por un especialista de Prohvilla, de acuerdo con la claridad, pertinencia y relevancia de la temática ambiental. Para la confiabilidad de las encuestas se realizó una prueba piloto con 5 empresas y negocios aplicando la prueba de KR 20, con el fin de ver la consistencia de las preguntas. Se obtuvo un valor de 0,75 para ambas encuestas, que supera lo mínimo aceptable $(0,70)$. Por ello, el instrumento se considera confiable para su aplicación en la muestra de estudio y se tuvo como variable.

\section{Métodos}

Durante la primera salida de campo realizamos observaciones del área en su totalidad, con la finalidad de identificar los impactos que probablemente son generados por las empresas y negocios. De igual modo, se realizaron fotografías y apuntes con el propósito de completar la investigación. Para obtener los datos, se llevó a cabo una segunda visita y se realizó la encuesta con 4 preguntas representativas a los negocios y empresas aledaños a la RVSPV. Con pocas de ellas no se pudo desarrollar la entrevista, de modo que se les envió la encuesta virtualmente.

Se analizaron las observaciones de campo y encuestas, y se utilizó el programa Excel para la elaboración de las figuras y cuadros. El RUC sirvió eventualmente para verificar e identificar las empresas o los negocios formales e informales a través de la página de la Superintendencia Nacional de Aduanas y de Administración Tributaria (Sunat, https://e-consultaruc.sunat.gob.pe/cl-tiitmrconsruc/FrameCriterioBusquedaMovil.jsp).

\section{a. Etapa de precampo}

En esta etapa se revisó información disponible de buscadores como Google Académico y repositorios como ScienceDirect, Redalyc, entre otros, y en páginas de instituciones estatales como la Sunat, el Servicio Nacional de Áreas Naturales Protegidas por el Estado (Sernanp) y otras afines. Esto se realizó para recopilar información con base en la investigación y respecto al área de estudio.

La tabla 2 presenta la lista de repositorios y páginas de instituciones revisadas por los autores.

Tabla 2. Lista de repositorios y páginas de instituciones revisados.

\begin{tabular}{|c|c|c|}
\hline $\begin{array}{l}\text { Repositorios, } \\
\text { revistas e } \\
\text { instituciones } \\
\text { revisados }\end{array}$ & $\begin{array}{c}\text { Temas de } \\
\text { investigación }\end{array}$ & URL \\
\hline $\begin{array}{l}\text { Universidad } \\
\text { Científica del } \\
\text { Sur }\end{array}$ & $\begin{array}{l}\text { Análisis del control } \\
\text { de emisiones } \\
\text { atmosféricas } \\
\text { vehiculares en Lima } \\
\text { Metropolitana }\end{array}$ & $\begin{array}{c}\text { https://revistas.cientifica. } \\
\text { edu.pe/index.php/ } \\
\text { southsustainability/article/ } \\
\text { view/598 }\end{array}$ \\
\hline Sernanp & Los Pantanos de Villa & $\begin{array}{c}\text { https://www.sernanp.gob.pe/ } \\
\text { los-pantanos-de-villa }\end{array}$ \\
\hline Redalyc & $\begin{array}{c}\text { Diagnóstico } \\
\text { ambiental en la } \\
\text { unidad empresarial } \\
\text { de base taller de } \\
\text { equipos }\end{array}$ & $\begin{array}{c}\text { https://www.redalyc.org/ } \\
\text { articulo.oa?id=1813330320045 }\end{array}$ \\
\hline Redalyc & $\begin{array}{c}\text { Diagnóstico } \\
\text { ambiental preliminar } \\
\text { y oportunidades de } \\
\text { prevención de la } \\
\text { contaminación en la } \\
\text { fábrica de helados } \\
\text { Mayarí }\end{array}$ & $\begin{array}{c}\text { https://www.redalyc.org/ } \\
\text { journal/4455/445558836008/ }\end{array}$ \\
\hline $\begin{array}{l}\text { Universidad } \\
\text { Sergio } \\
\text { Arboleda }\end{array}$ & $\begin{array}{c}\text { Responsabilidad } \\
\text { ambiental en las } \\
\text { empresas que afectan } \\
\text { la atmósfera }\end{array}$ & $\begin{array}{l}\text { https://revistas. } \\
\text { usergioarboleda.edu.co/index. } \\
\text { php/visiuris/article/view/1143 }\end{array}$ \\
\hline
\end{tabular}




\begin{tabular}{ccc}
\hline $\begin{array}{c}\text { Revista } \\
\text { Espacios }\end{array}$ & $\begin{array}{c}\text { Análisis de los } \\
\text { elementos implícitos } \\
\text { en la validación de } \\
\text { contenido de un } \\
\text { instrumento de } \\
\text { investigación }\end{array}$ & $\begin{array}{c}\text { http://www.revistaespacios. } \\
\text { com/cited2017/cited2017-23. } \\
\text { pdf }\end{array}$ \\
& Ley 28611, Ley & https://www.minam. \\
Minam & gob.pe/wp-content/ \\
& Ambiente & uploads/2017/04/Ley- \\
& N\%C2\%BO-28611.pdf \\
\hline
\end{tabular}

\section{b. Etapa de campo}

Se realizaron dos salidas de campo. Durante la primera se llevó a cabo el reconocimiento de la urbanización Huertos de Villa mediante observación, con la finalidad de identificar los posibles impactos ambientales generados y la cantidad de empresas y negocios (población). La muestra fue por conveniencia con 6 empresas y 7 negocios, y fueron reconocidas a favor de los autores (los que accedieron a desarrollar la encuesta). Además, se realizó la toma de fotografías y apuntes para completar la investigación. Para obtener los datos de la encuesta, se ejecutó una segunda visita a las empresas (gerentes o encargados) y los negocios (propietarios) aledaños al RVSPV a través de formularios. No fue posible adquirir información en algunas empresas y, por ello, se les envió la encuesta a sus correos.

\section{c. Etapa de gabinete}

Se analizó la información recolectada a partir de la observación de campo. Se utilizó el programa Microsoft Excel 2016 para la elaboración de las figuras y los cuadros de las encuestas realizadas. También se empleó el programa ArcGis 10.5 para ubicar espacialmente las empresas y los negocios situados en el área de estudio.

\section{Resultados}

En la zona de estudio se identificó un total de 16 empresas y 9 negocios. Con el fin de constatar si eran formales o informales, se usaron los RUC proporcionados

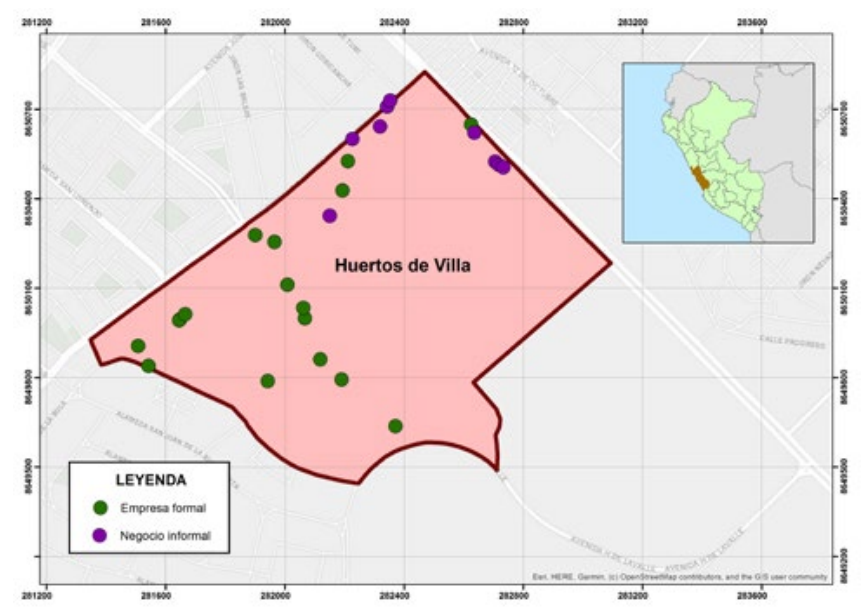

Figura 2. Ubicación de las empresas y los negocios.

Fuente: Elaboración propia. en la encuesta mediante la página de la Sunat. En el caso de los no encuestados se buscaron los datos generales y se realizó el mismo procedimiento. Se determinó que las empresas en su totalidad eran formales, mientras que los negocios eran todos informales.

Para la investigación se trabajó con una muestra por conveniencia, sobre la base de las empresas y los negocios que accedieron a realizar la encuesta (6 empresas y 7 negocios). A continuación, se muestran las preguntas realizadas en las encuestas (ver tablas 3 y 4 ).

\section{a) Encuesta aplicada a las empresas}

Tabla 3. Nivel de respuesta: encuesta a las empresas.

\begin{tabular}{|c|c|c|c|}
\hline \multirow{2}{*}{\multicolumn{2}{|c|}{ Preguntas de la encuesta }} & \multicolumn{2}{|c|}{$\begin{array}{c}\text { Porcentaje } \\
(\%) \\
\end{array}$} \\
\hline & & Sí & No \\
\hline 1. & $\begin{array}{l}\text { ¿La empresa cuenta con contenedores de } \\
\text { residuos? }\end{array}$ & 100 & 0 \\
\hline 2. & $\begin{array}{l}\text { ¿La empresa segrega los residuos sólidos para su } \\
\text { posterior reciclaje? }\end{array}$ & 100 & - \\
\hline 3. & $\begin{array}{l}\text { ¿La empresa realiza capacitaciones a su personal } \\
\text { relacionadas con temas de manejo de residuos } \\
\text { sólidos? }\end{array}$ & 100 & 0 \\
\hline 4. & $\begin{array}{l}\text { ¿La empresa utiliza materia prima que favorece al } \\
\text { medioambiente? }\end{array}$ & 50 & 50 \\
\hline 5. & $\begin{array}{l}\text { ¿La empresa realiza periódicamente el } \\
\text { mantenimiento a los equipos y maquinarias de los } \\
\text { procesos productivos? }\end{array}$ & 67 & 33 \\
\hline 6. & $\begin{array}{l}\text { ¿La empresa realiza recirculación de aguas en sus } \\
\text { procesos productivos? }\end{array}$ & 17 & 83 \\
\hline 7. & $\begin{array}{l}\text { ¿La empresa realiza el tratamiento previo de sus } \\
\text { aguas residuales antes del vertimiento a la red de } \\
\text { alcantarillado? }\end{array}$ & 17 & 83 \\
\hline 8. & $\begin{array}{l}\text { ¿Su empresa cuenta con una oficina que realice } \\
\text { actividades de gestión ambiental? }\end{array}$ & 17 & 83 \\
\hline & $\begin{array}{l}\text { ¿La empresa cuenta con un instrumento de } \\
\text { gestión ambiental? }\end{array}$ & 33 & 67 \\
\hline & $\begin{array}{l}\text { ¿Estaría dispuesto a implementar algún } \\
\text { instrumento de gestión ambiental a su empresa? }\end{array}$ & 100 & $0^{1}$ \\
\hline
\end{tabular}

Los resultados de las encuestas (figura 3) indicaron que el $100 \%$ de las empresas cuentan con contenedores para disponer y segregar residuos sólidos por tipo, realizan capacitaciones a su personal en temática ambiental, y estarían dispuestos a implementar un instrumento de gestión ambiental, a pesar de que solo el $33 \%$ cuenta con ello. Asimismo, el $67 \%$ realiza mantenimiento a los equipos y maquinarias de los procesos productivos, y existe una gran preocupación en el manejo de aguas residuales, debido a que solo el $17 \%$ realiza un previo tratamiento antes del vertimiento a la red de alcantarillado.

\footnotetext{
$\overline{1 \text { Porcentaje del total }}$ de negocios encuestados.
} 
Empresas que cuentan con contenedores

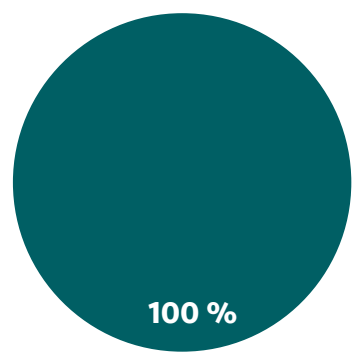

Empresas que realizan capacitaciones a su personal sobre temas de manejo de residuos

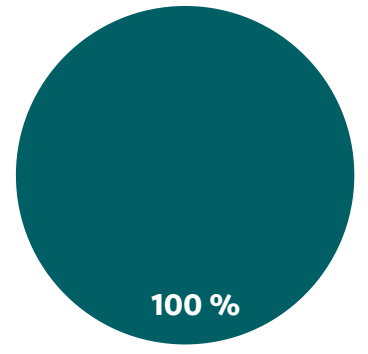

Empresas que realizan previo tratamiento de sus aguas residuales antes del vertimiento a la red de alcantarillado

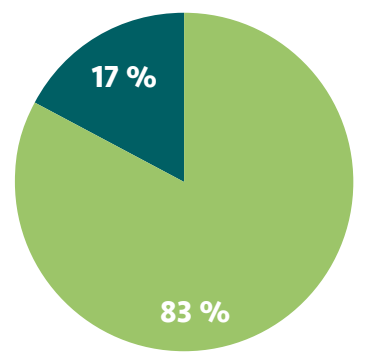

Cantidad de empresas que cuentan con un instrumento de gestión ambiental

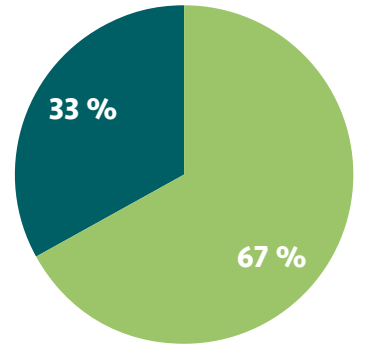

Cantidad de empresas que realizan mantenimiento a los equipos y maquinarias

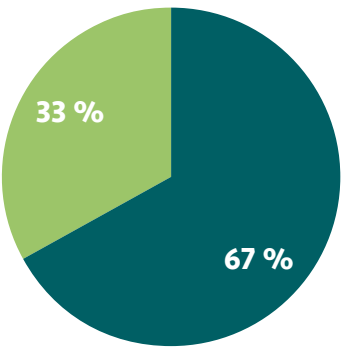

Cantidad de empresas que segregan los residuos para un posterior reciclaje

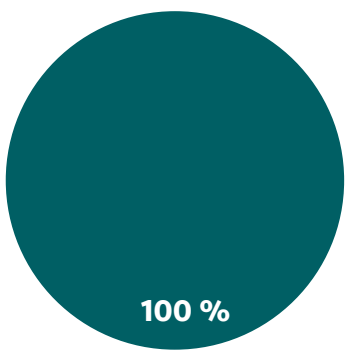

Empresas que utilizan materia prima que favorece al medioambiente

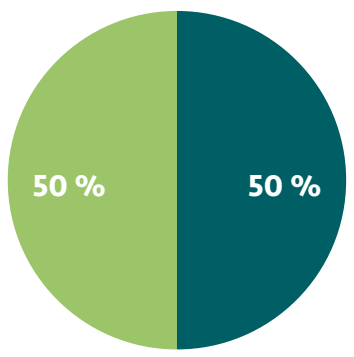

Cantidad de empresas que cuentan con una oficina de gestión ambiental

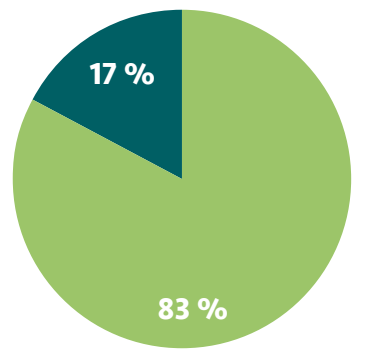

Empresas dispuestas a implementar un instrumento de gestión ambiental

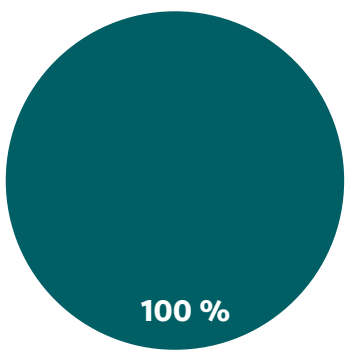

Empresas que realizan recirculación de aguas en sus procesos productivos

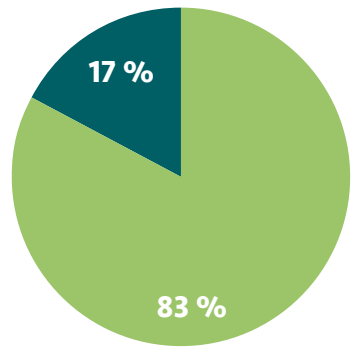

Figura 3. Resultados obtenidos respecto a las empresas evaluadas. 


\section{b) En los negocios}

Tabla 4. Nivel de respuesta: encuesta a los negocios.

\begin{tabular}{|c|c|c|}
\hline \multirow[t]{2}{*}{ Preguntas de la encuesta } & \multicolumn{2}{|c|}{$\begin{array}{l}\text { Porcentaje } \\
(\%)\end{array}$} \\
\hline & Sí & No \\
\hline 1. ¿Su negocio cuenta con recipientes de residuos? & 29 & 71 \\
\hline $\begin{array}{l}\text { 2. ¿El negocio segrega los residuos sólidos para su } \\
\text { posterior reciclaje? }\end{array}$ & 14 & 86 \\
\hline $\begin{array}{l}\text { 3. ¿El negocio reutiliza los recipientes de los productos } \\
\text { para generar un subproducto? }\end{array}$ & 29 & 71 \\
\hline $\begin{array}{l}\text { 4. ¿Utiliza algún método de ahorro de agua en su } \\
\text { negocio? }\end{array}$ & 29 & 71 \\
\hline $\begin{array}{l}\text { 5. ¿Considera que hace un uso racional y eficiente del } \\
\text { agua? }\end{array}$ & 43 & 57 \\
\hline $\begin{array}{l}\text { 6. ¿Ha presenciado a pobladores vertiendo residuos a } \\
\text { los canales? }\end{array}$ & 29 & 71 \\
\hline $\begin{array}{l}\text { 7. ¿Cree que sus actividades benefician al } \\
\text { medioambiente? }\end{array}$ & 14 & 86 \\
\hline $\begin{array}{l}\text { 8. ¿Le gustaría participar en una campaña para el } \\
\text { cuidado del medioambiente? }\end{array}$ & 29 & $71^{2}$ \\
\hline
\end{tabular}

De la encuesta aplicada a los negocios (figura 4), se obtuvo como resultado que solo el $14 \%$ de los negocios segregan sus residuos para posteriormente realizar su reciclaje. Dicho porcentaje es lamentable, ya que disponen cantidades de residuos sólidos que podrían ser reaprovechados, y al mismo tiempo es preocupante, porque los residuos generados podrían estar recibiendo una disposición inadecuada. Respecto al uso racional y eficiente del agua, resultó que el 43 \% realiza esa acción; sin embargo, el resultado difiere con lo observado en campo, ya que en 5 negocios evaluados se visualizaron prácticas inadecuadas del uso de agua durante sus actividades, porque utilizaban mangueras y baldes para lavar vehículos o limpiar sus áreas de trabajo.

\section{Discusión}

De acuerdo con los resultados obtenidos por las empresas, se demostró que solo el 33 \% cuenta con un instrumento de gestión ambiental. Este porcentaje es bajo con respecto al $100 \%$, ya que las actividades que realizan dentro o fuera de la organización podrían estar afectando al medioambiente. De manera semejante, Acuña et al. (2017) señalan que las empresas «no actúan de manera correcta sobre los factores ambientales, debido a que priorizan el rebajar los costos y riesgos que puedan repercutir más adelante en sanciones, en inversiones y reparaciones económicas», dejando de lado el sentido ambiental. Asimismo, manifiestan que los aspectos ambientales no son prioridad única del departamento de

$\overline{2 \text { Porcentaje del total }}$ de negocios encuestados. medioambiente, sino de todas las áreas de una empresa. Como se sabe, implementar un instrumento de gestión ambiental contribuye en el manejo, la prevención y la disminución de los impactos ambientales.

Por otra parte, se obtuvo como resultado que solo el $17 \%$ de las empresas evaluadas (es decir, una organización) realiza el previo tratamiento de sus aguas residuales antes del vertimiento a la red de alcantarillado, lo cual resulta preocupante, ya que las aguas residuales podrían tener exceso de concentración de los parámetros y, de esta manera, impactar negativamente al ambiente. El Decreto Supremo 010-2019-VIVIENDA, «Reglamento de Valores Máximos Admisibles (VMA) para las descargas de aguas residuales no domésticas en el sistema de alcantarillado sanitario», indica, en su artículo 8 del capítulo II, que las unidades que descargan aguas residuales no domésticas deben implementar un sistema de tratamiento de aguas residuales y/o las modificaciones del proceso productivo cuando sus descargas exceden los VMA. Asimismo, deben cumplir con las normas sectoriales que se emitan para regular la aplicación de los VMA.

Además, durante la presente investigación se presentaron algunas limitaciones, como no obtener respuesta de todas las empresas consideradas para el estudio, ya que se negaron a ser encuestadas o no respondieron el link del formulario enviado a los correos proporcionados, mostrando poco interés y participación en el estudio. Se percibió en campo que esta reacción probablemente se debió a que las industrias tienen un mal concepto de la supervisión de las autoridades, ya que creían que iban a ser sancionadas. Por ello, Magaña et al. (2019) indicaron que es necesaria una evaluación del impacto ambiental, y así visualizar los principales impactos ambientales y plantear oportunidades para prevenir la contaminación que genera.

Por otro lado, a partir del presente diagnóstico se evidenció una gran deficiencia y falta de interés en el tema ambiental por parte de las empresas y los negocios. Sánchez et al. (2014) señalan que un diagnóstico ambiental es una herramienta importante para el establecimiento de metas y objetivos ambientales, ya que ayudan a plantear medidas de mitigación de los impactos ambientales detectados.

\section{Conclusiones}

- De las empresas encuestadas se identificó que solo dos de ellas presentan un instrumento de gestión ambiental, es decir, que solamente el $33 \%$ de las empresas formales cuentan con un plan de gestión ambiental en la urbanización Huertos de Villa.

- Se identificó la presencia de un total de 16 empresas formales y 9 negocios informales en la urbanización Huertos de Villa adyacente del área natural protegida RVSPV.

- Las empresas, en su gran mayoría, mostraron una actitud no participativa y limitaban el acceso para el desarrollo 


\section{Cantidad de negocios que cuentan con recipientes de residuos}

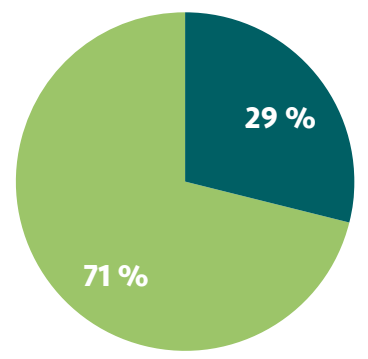

Negocios que benefician con sus actividades al medioambiente

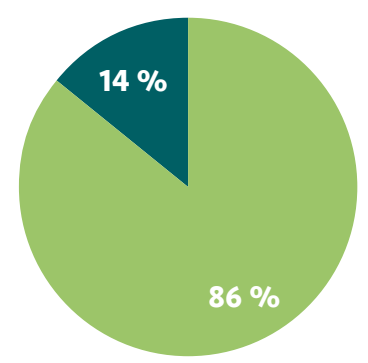

Negocios que consideran que hacen un uso racional del agua

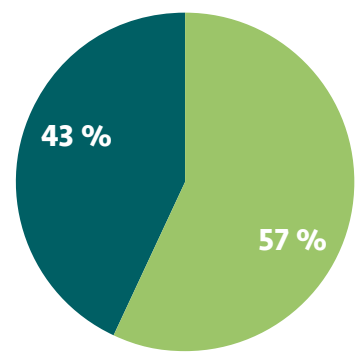

Negocios que reutilizan los recipientes de los productos para generar un subproducto

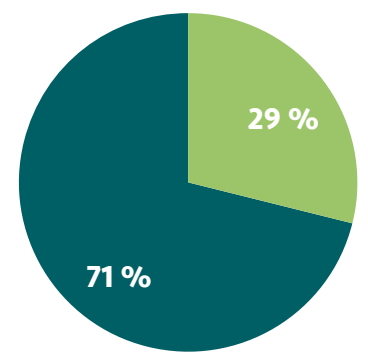

\section{Cantidad de negocios que segregan los residuos para un posterior reciclaje}

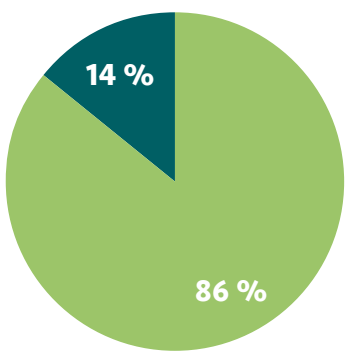

Negocios que participarían en campañas sobre el cuidado del medioambiente

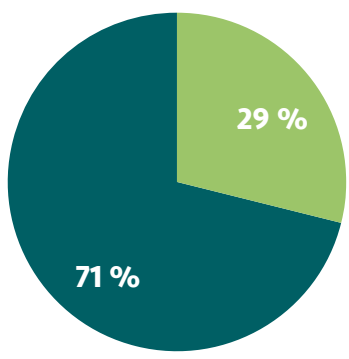

Negocio que presenciaron el vertimiento de residuos en los canales

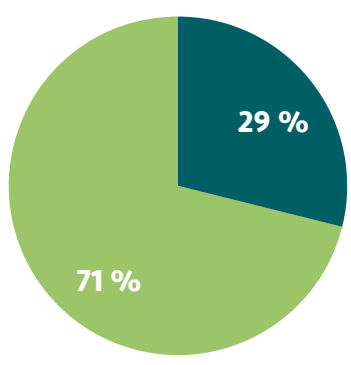

Negocios que utilizan algún método de ahorro de agua

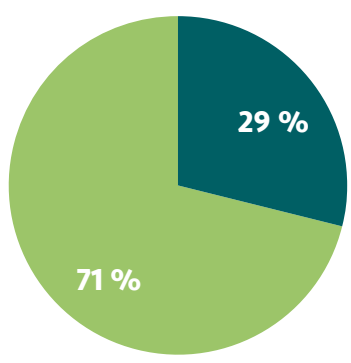

No

Figura 4. Resultados obtenidos respecto a los negocios evaluados.

de las encuestas, probablemente debido al temor de ser supervisadas por alguna autoridad o entidad fiscalizadora.

- De acuerdo con los resultados obtenidos en las encuestas, se identificó que algunos negocios, a pesar de no tener un instrumento de gestión ambiental, incorporan en sus actividades buenas prácticas ambientales. Así, el $71 \%$ señaló que reutilizan productos para generar un subproducto.

\section{Recomendaciones}

- Brindar sensibilización ambiental para las empresas y los negocios ubicados en la urbanización Huertos de Villa, con el fin de promover buenas prácticas ambientales y evitar o minimizar impactos negativos en el ambiente, así como posibles impactos en el RVSPV. 
- Complementar con estudios posteriores la evaluación de los posibles impactos ambientales (generación de ruido y afectaciones en la calidad del aire) que se están generando como consecuencia de las actividades realizadas. Asimismo, identificar los aspectos ambientales que estarían generando las empresas y los negocios, como residuos sólidos, aguas residuales, etc., y cómo realizan su disposición o tratamiento.

- De esta manera, se puede contribuir con brindar información base a los tomadores de decisiones (por ejemplo, autoridades $\mathrm{u}$ otras partes interesadas), para que puedan determinar acciones concretas de cara a identificar oportunidades de mejora en la gestión ambiental en la zona de estudio.

\section{Agradecimientos}

Los autores agradecen el apoyo del jefe de Área de Ciudadanía Ambiental Carlos Quispe Sarria, que estuvo presente durante el proceso de construcción del artículo, así como a Dámaso W. Ramírez, quien brindó asesoramiento en la metodología de investigación.

\section{Fuente de financiamiento}

Esta investigación tuvo el soporte logístico de Prohvilla.

\section{Contribución de autoría}

JLN-F y JA-L contribuyeron en la planificación de la investigación. JLN-F, JA-L y SY-Q contribuyeron en la ejecución, el procesamiento de datos y el análisis de los resultados y la redacción. Todos los autores aprobaron la versión final del texto.

\section{Potenciales conflicto de interés}

Los autores declaran que no existe ningún conflicto de interés.

\section{Referencias bibliográficas}

Aguilar-Galvis, G., Banquet-Suárez, A. y Falquez O’Byrne, A. (2017). «Responsabilidad ambiental en las empresas que afectan a la atmósfera». Vis luris, 4(7), pp. 50-71. Disponible en: https://doi. org/10.22518/vis.v4i72017.1143

Gobierno del Perú. (11 de marzo de 2019). Decreto Supremo 010-2019-VIVIENDA, Reglamento de Valores Máximos Admisibles (VMA) para las descargas de aguas residuales no domésticas en el sistema de alcantarillado sanitario. Disponible en: https://cdn.www. gob.pe/uploads/document/file/306588/DS_010-2019-VIVIENDA. pdf

Juárez, L. y Tobón, S. (2018). «Análisis de elementos implícitos en la validación de contenido de un instrumento de investigación». Revista Espacios, 39(53).

He, Z., Cheng, X., Kyzas, G. Z. y Fu, J. (2016). «Pharmaceuticals pollution of aquaculture and its management in China». Journal of Molecular Liquids, 223, pp. 781-789.
León Sulca, G. M. (2019). «Gobernanza ambiental y conservación: las gestiones del SERNANP y PROHVILLA en el Refugio de Vida Silvestre los Pantanos de Villa, y las acciones para su conservación». Argumentos, 1, pp. 119-124.

Olaussen, J. O. (2018). «Environmental problems and regulation in the aquaculture industry. Insights from Norway». Marine Policy, 98, pp. 158-163.

Magaña-Irons, L González-Díaz, Y Nápoles-Meléndez, L. y Ojeda Armaignac, E. (2019). «Diagnóstico ambiental preliminar y oportunidades de prevención de la contaminación en la Fábrica de Helados Mayarí. Cuba». Tecnología Química, 39(1), pp. 105-116.

Pacheco Bustos, C. Fuentes Pumarejo, L. G Sánchez Cotte, É. H. y Rondón Quintana, H. A. (2017). «Residuos de construcción y demolición (RCD), una perspectiva de aprovechamiento para la ciudad de Barranquilla desde su modelo de gestión». Ingeniería y Desarrollo, 35(2), pp. 533-555.

Pulido, V. (2018). «Ciento quince años de registros de aves en Pantanos de Villa». Revista Peruana de Biología, 25(3), pp. 291-306.

Ramirez, D. W., Aponte, H., Lertora, G. y Gil, F. (2018). «Incendios en el humedal Ramsar Los Pantanos de Villa (Lima-Perú): Avances en su conocimiento y perspectivas futuras». Revista de Investigaciones Altoandinas, 20(3), pp. 347-360.

Sánchez-Vera, Z., Sánchez-Paz, H. R. y Carballo-Vázquez, A. R. (2014). «Diagnóstico ambiental en la unidad empresarial de base taller de equipos». Ciencia en su PC, (3), pp. 42-57.

Solano, P. y Chirinos, C. (2001). «Lucchetti vs los Pantanos de Villa: un análisis legal». Disponible en: https://spda.org.pe/?wpfb_dl=51 